\section{$\underset{\substack{\text { hommes } \\ \text { \& migrations }}}{ }$}

\section{Hommes \& migrations}

Revue française de référence sur les dynamiques

migratoires

1312 | 2015

Diasporas iraniennes

\title{
Histoire de photos
}

\section{Mustapha Harzoune}

\section{(2) OpenEdition}

Journals

\section{Édition électronique}

URL : http://journals.openedition.org/hommesmigrations/3534

DOI : 10.4000/hommesmigrations.3534

ISSN : 2262-3353

\section{Éditeur}

Musée national de l'histoire de l'immigration

\section{Édition imprimée}

Date de publication : 1 octobre 2015

Pagination : 146-147

ISBN : 978-2-919040-33-9

ISSN : $1142-852 X$

\section{Référence électronique}

Mustapha Harzoune, «Histoire de photos », Hommes \& migrations [En ligne], 1312 | 2015, mis en ligne le 31 mai 2016, consulté le 14 septembre 2020. URL : http://journals.openedition.org/

hommesmigrations/3534 


\section{KIOSQUE}

\section{HISTOIRE DE PHOTOS}

par MUSTAPHA HARZOUNE.

a rentrée semble avoir prolongé les vacances Lestivales. Histoire de photos et de plage ! Le temps de plier serviettes et parasols, et le sable encore chaud des corps alanguis, lubrifiés à la crème solaire, a accueilli, malgré lui, d'autres corps. Un autre corps. Celui d'un enfant de trois ans. Sans vie. Froid. Retrouvé mort le 2 septembre 2015 sur une plage turque. Le visage planté dans l'obscurité minérale, tournant le dos à la lumière du soleil, à d'autres réservée. II s'appelait Aylan, et, le temps de l'émotion, il est devenu l'enfant de tous et de chacun. Un déchirement ? Sûrement. Un déclic ? Peut-être.

Pour Widad Kefti (Bondy Blog), la photo "raconte tout de notre honteuse lâcheté, de notre totale irresponsabilité, de notre misérable échec. Un bébé victime de notre société égoïste, froide, folle, qui croit pouvoir gérer une crise humanitaire comme elle gère un nid de cafards. Un bébé échoué sur une plage, face contre terre, à l'image de nos consciences, échouées, mortes noyées avec lui. 'Si cette photo puissante ne change pas le regard de l'Europe, qu'est-ce qui le fera?' se demandait The Independant. 'Si nous n'exigeons pas de nos gouvernements les mesures extrêmes qu'impose cette urgence humanitaire, qui le fera?"'

Pourtant, l'album médiatique refermé, on est passé à autre chose. Oubliant que depuis le 2 septembre, plus d'une centaine d'enfants auraient trouvé la mort en mer d'Égée. Comme Aylan. Mais, désormais, "dans une indifférence quasi générale" (Libération, 5 novembre).

Depuis, d'autres photos s'emparent des esprits, reconfigurent la carte de nos affects; celles de milliers d'hommes et de femmes qui marchent sur les routes européennes, comme un trop-plein que le Proche-Orient, I'Afrique ou l'Asie, ne peut plus contenir. Point de théorie des fluides ici, mais un rappel : I'humanité est une et la terre un partage. Le droit de vivre n'importe où versus le vertige de l'enracinement avancé comme un acte de propriété. Alors, ces frères en humanité font ce que d'autres ont fait avant eux. Parfois violemment. Ils marchent. Pacifiquement. Non en porteurs de mort, mais en messagers de vie."Il est plus qu'urgent de comprendre que nous sommes liés à ces réfugiés, ces êtres humains qui abandonnent tout ce qu'ils sont, tout ce qu'ils possèdent, pour tenter de survivre; liés par le sang. Ils sont les héritiers de nos échecs successifs. Inutile de se cacher derrière des idées populistes pour camoufler une politique criminelle. (...) Nous n'avons pas d'autre choix que d'accueillir les vivants ou toutes nos vies seront hantées par leur mort. À tout jamais" (Widad Kefti). Le corps d'Aylan a laissé place aux longues files de réfugiés, aux camps et aux barbelés derrière lesquels sont retenus, internés, concentrés hommes, femmes et enfants. II y a les images et les mots qui vont avec, "déferlement" ou "invasion". D'une image l'autre, d'une émotion l'autre : à la compassion et la solidarité succèdent la peur et le repli.

Pourtant, cette photo d'Aylan peut-elle aider à recomposer du politique ? demande Guillaume Erner à Frédéric Lordon, économiste et philosophe : "Assurément... Les idées ne deviennent véritablement efficaces au sens de capacité à produire des effets, que si elles sont accompagnées d'affects. (...) Toute la philosophie de Spinoza est consacrée à lutter contre ce qu'il appelle l'impuissance de la raison, qu'il énonce dans cette proposition à mes yeux décisive de l'Éthique qui dit : 'La connaissance vraie du bien et du mal ne saurait réprimer aucun affect en tant qu'elle est une connaissance vraie.' Une phrase que Bourdieu a reproduite pour son propre 
compte en disant qu"il n'y a pas de force intrinsèque des idées vraies.' Et en effet, il faut le secours des affects, en l'occurrence produits par une image, pour donner de la puissance et de l'efficacité aux idées, c'est-à-dire pour leur permettre de déterminer les corps à se mettre en mouvement" (France Culture, le 23 septembre).

\section{"Déclic"...}

De fait, après la diffusion de la photo d'Aylan, l'opinion se serait retournée "contre Sarkozy et Marine Le Pen", écrit Bruno Roger-Petit (challenges.fr, 11 septembre) : "Lopinion est passée de l'hostilité envers l'accueil des réfugiés à la bienveillance. Estce le signe que la droitisation des Français n'est pas un phénomène irréversible? Peu à peu, le mot 'réfugié' remplace celui de 'migrants'. II n'y a guère plus que Nicolas Sarkozy (et ses soutiens), Marine Le Pen (et ses soutiens), Éric Zemmour (et ses soutiens) et quelques autres encore, qui répugnent à nommer comme tels ceux qui méritent de l'être, leur refusant cette part de dignité qui confère l'humanité." Et de citer un sondage (Elabe/BFMTV) qui annonce "qu'une majorité de Français se montrait désormais favorable à l'accueil des réfugiés (...). II aura suffi d'une photo et d'un mot, 'réfugié,' enfin employé à bon escient, pour bouger les consciences". "C'est le camp du progrès humain qui l'emporte, écrit le journaliste. Ce bouleversement, dicté par une émotion légitime, (...) est finalement rassurant pour les républicains de tous bords." II serait "annonciateur d'un vent favorable. La question est désormais de savoir si ce noyau dur [une frange de l'électorat "Les Républicains" et celui du FN] peut être électoralement majoritaire au regard des enjeux politiques qui seront au coeur de la prochaine campagne présidentielle".

Christophe Barbier écrit : contre la crise identitaire, accueillons plus de migrants", et prône une politique d'“intégration par le bas": "Notre nation ne s'est construite et renforcée qu'en mêlant, venus de tous les horizons, ceux qui adhéraient à ses valeurs.
Puisque les migrants fuient la dictature de Bachar et la barbarie de Daech, leur place est ici, comme l'était celle des boat people asiatiques il y a quarante ans. Et si l'on veut qu'ils les adoptent, respectons nousmêmes nos valeurs républicaines, à commencer par cette hospitalité tout hugolienne due aux damnés de la terre. Au gouvernement de favoriser un tel état d'esprit, non en promettant aux étrangers un utopique droit de vote, mais en soutenant les associations et les clubs de sport qui garantissent l'intégration par le bas" (L'Express, 29 septembre).

Au lieu d'opposer les souffrances, le Mouvement national des chômeurs et précaires a, le 10 septembre, répondu à Mme Le Pen qui déclarait que l'accueil des réfugiés était "un crachat au visage des chômeurs français et européens": "Ce sont vos mots et vos idées qui sont un crachat au visage des chômeurs. (...) Les chômeurs et précaires sont des citoyens libres de leurs idées et de leur vote. Mais il est certain que leur précarité ne saurait les égarer dans les marais malsains de l'extrême droite. Le repli sur soi, le rejet de l'Autre, l'individualisme égoïste et la haine sociale ne seront jamais l'attitude des chômeurs et des précaires, ni de leurs organisations collectives" (www.mncp.fr).

Dans La République des Pyrénées (4 septembre), Jean-Michel Helvig écrit que I'“image sacrificielle (...) soulève une émotion qui impose même silence à ceux qui cadenassent leurs coeurs et leurs frontières. Que cette photo puisse à elle seule provoquer le déclic qui va hisser l'Union européenne au niveau de ses responsabilités historiques, ce serait le plus bel hommage à rendre à la mémoire d'Alyan Kurdi (...)". Mais, comme un bémol à ce qui précède, tombe la question: "Mais de quel 'déclic'cette Europe est-elle capable? Angela Merkel et François Hollande veulent taper du poing sur la table pour que l'Union adopte une 'répartition' équitable mais surtout 'contraignante' des réfugiés. C'est revenir à cette idée de quotas rejetée avant l'été, notamment par François Hollande qui s'abritait derrière le droit d'asile. Justement ce robinet-là a été à peine desserré et, au demeurant il n'y a pas d'harmonisation des critères d'asile parmi les 28 États membres. La 


\section{KIOSQUE}

France et l'Allemagne pourront-elles imposer leurs vues à une Hongrie où l'on érige un mur de barbelés pour empêcher 'l'invasion', à une Slovaquie qui ne veut accueillir que des 'chrétiens' ou encore au Royaume-Uni qui se barricade dans son île?"

\section{... et couacs}

Tandis que Romaric Godin explique "pourquoi Angela Merkel est si généreuse envers les réfugiés" (La Tribune, 7 septembre), le 11 septembre, Patrick Devedjian croit faire de l'humour : "Les Allemands nous ont pris nos juifs, ils nous rendent des Arabes." Si le député des Hauts-de-Seine s'est excusé illico, Nadine Morano avance, elle, franco : "Je n'ai pas envie que la France devienne musulmane. (...) Ce ne serait plus la France (...). La France est un pays aux racines judéo-chrétiennes, la France est un pays de race blanche" (France 2, On n'est pas couché, le 26 septembre). À ce niveau, il faut lire la "Lettre ouverte à un ex-ami” (Les invités de Médiapart, 29 septembre), de Shlomo Sand en réponse à une tribune de Pierre-André Taguieff publiée dans Le Monde. l'historien israélien écrit : "Tu sais bien que la haine envers celui qui est un peu différent, et que l'imaginaire apeuré face à celui qui affiche une singularité, ne se limitent pas aux émotions stupides de gens incultes, situés au bas de l'échelle sociale. Tu sais bien que cela n'épargne pas les classes sociales bien éduquées. Durant la période tragique pour les juifs et leurs descendants (1850-1950), le langage judéophobe ne se donnait pas uniquement libre cours dans les faubourgs populaires, mais il s'exprimait aussi dans la haute littérature, dans la philosophie raffinée et dans la grande presse. La haine et la peur des juifs faisaient partie intégrale des codes culturels, dans toutes les couches de la société européenne. Cet état de fait s'est, fort heureusement, modifié dans les années qui ont suivi la fin de la Seconde Guerre mondiale." Aujourd'hui, poursuit l'historien, "la judéophobie a perdu toute légiti- mité dans l'espace public. (...) Le prix à payer pour la disparition de cette 'belle haine' (pour user d'un qualificatif de l'antisémitisme en vogue il y a un siècle), fut, comme l'on sait, très élevé. De nos jours, la 'belle haine' est effectivement tournée vers d'autres gens, et nous ne savons pas encore quel en sera le prix. Faut-il, comme tu t'y emploies également, nier que l'islamophobie a, effectivement, remplacé la judéophobie, et jouit d'une légitimité croissante dans tous les secteurs culturels français?".

Dans La Croix (17 septembre), Patrick Boucheron rappelle qu'en 1979, "130 ooo réfugiés vietnamiens et cambodgiens ont été accueillis en France en très peu de temps (...). Que s'est-il passé entre cette époque et aujourd'hui, pour que l'autre rive de la Méditerranée nous semble plus éloignée de nous, ou plus menaçante, que ne l'était le golfe de Siam? Pas seulement l'augmentation du chômage, mais trente ans de combat idéologique désignant l'antiracisme et, au-delà, l'ouverture à l'autre, comme le principal danger qui menace l'identité nationale. Voyez quel cynisme, et surtout quelle conception étriquée des grandeurs d'une nation tout cela produit: un mélange détonant d'arrogance et d'abandon, une perte de confiance dans les ressources morales d'une société. Au fond, dans ce procès permanent fait à la pensée critique des années 1970, c'est le lien à l'universel qui se trouve en danger". Avec Jürgen Habermas, il rappelle que l'hospitalité est un droit et que "ce droit n'est pas soumis à la discussion politique. Ne faisons pas comme si c'était un sujet de débat". Mais "il ne faut pas faire les belles âmes, se contenter d'un vague 'N'ayez pas peur' - pendant que les gouvernements, eux, ne cessent d'avoir peur de leurs opinions publiques qu'ils supposent hostiles aux étrangers, au point de les conformer comme telles. II ne s'agit pas de nier la peur mais de l'affronter pour mieux la réorienter vers les menaces véritables. 24000 réfugiés ne menacent ni l'identité, ni la sécurité, ni la prospérité de la France - mais il y a de quoi avoir 
peur, vraiment peur, d'un pays qui ne se sentirait pas ou plus capable de les accueillir".

Pour François Bonnet, "la réponse française est à ce jour d'une indigne médiocrité". "François Hollande a désormais formulé une politique: quotas d'accueil de réfugiés par pays; harmonisation des droits d'asile ; création en Grèce et en Italie de 'hotspots', centres d'accueil où seraient triés demandeurs d'asile et migrants économiques. Et le président a donné un chiffre : la France est prête à accueillir 24000 réfugiés dans les deux ans (...), soit dix personnes par mois et par département..." Par la voix d'Angela Merkel qui plaide en faveur de l'accueil des réfugiés, l'Allemagne serait "en train d'accéder au rang de grande puissance, quand la France s'isole et se rétrécit dans des débats où la peur le dispute à l'égoïsme". Pourtant, "le président devait se livrer à cet exercice d'autoglorification qui ne cesse de faire sourire à l'étranger : 'C'est parce que nous sommes dépositaires de cette grande idée de progrès, j'allais dire de cette grande idée de la France pour le monde, que beaucoup de pays nous marquent leur solidarité et que beaucoup de peuples nous témoignent leur reconnaissance.' Le président avait-il alors conscience que cette France-là n'est plus ? Vingt ans de politique migratoire à double verrou, un ministère de l'Immigration et de l'Identité nationale, le discours de Dakar, les déploiements policiers de Bernard Cazeneuve et la fermeté revendiquée par Manuel Valls ont fait de la France un repoussoir, simple pays de transit vers d'autres eldorados rêvés, Royaume-Uni, Suède, Norvège, Allemagne. (...) C'est aussi ce désastre politique français que nous raconte aujourd'hui la crise des réfugiés" (Médiapart, 11 septembre).

\section{Autre focale et autre cliché}

Dans La paille et la poutre (9 septembre, leaders. com), Sophie Bessis élargit la focale : "Quelles que soient les réticences de ses classes politiques, la fri- losité de ses populations, les peurs d'une partie de ses opinions travaillées par les discours de l'exclusion, l'Europe donne aujourd'hui l'exemple en matière d'accueil." Car, sur la rive sud de la Méditerranée, "hormis le Liban et la Jordanie, (...) qui abritent bon gré mal gré des millions de réfugiés, aucun État arabe ayant aujourd'hui la chance d'être en paix n'a levé le petit doigt pour venir en aide aux 'frères' syriens et irakiens. Les richissimes monarchies du Golfe se fichent comme d'une guigne de la souffrance humaine (...). Où sont l'Égypte, la Tunisie, l'Algérie, le Maroc ? (...) Pas un rassemblement de solidarité, pas une proposition d'accueil même symbolique, pas une quête, pas un geste qui relèverait de la plus simple compassion (...)". Pire, "les mêmes" reprochent "à l'Occident de ne pas en faire assez, pendant qu'eux en font moins encore. Ils crient au racisme dont souffrent au nord du monde les populations immigrées, pendant que dans leurs pays les migrants subsahariens sont le plus souvent traités comme des sous-hommes et que beaucoup sont renvoyés chez eux sans ménagement. Ceux qui déplorent avec raison les milliers de noyés qui gisent au fond de la Méditerranée savent-ils que le Sahara est une mer de sable où meurent par centaines des Africains que l'Algérie - entre autres - expulse de son territoire?"

Loin de ces débats, une autre photo faisait le tour des réseaux sociaux, "le baiser des réfugiés syriens", un baiser échangé sur l'esplanade de la gare de Keleti à Budapest, "à l'abri d'une tente exiguë, semblant oublier le reste du monde, mettant de côté pour une seconde les atrocités qui les ont poussés à fuir leur pays, les discussions sans fin des dirigeants de l'Europe et le long voyage qui les attend encore, ils sont tournés l'un vers l'autre pour partager un moment de tendresse". "Comme une revanche, un message d'espoir", écrit Leïla Marchand (Slate.fr, le 22 septembre), avant de citer István, l'auteur du cliché : "L'amour est la seule chose qui transcende l'espace et le temps." 